\title{
Interaction-driven phases in the half-filled spinless honeycomb lattice from exact diagonalization
}

\author{
Noel A. García-Martínez, ${ }^{1}$ Adolfo G. Grushin, ${ }^{1}$ Titus Neupert, ${ }^{2}$ Belén Valenzuela, ${ }^{1}$ and Eduardo V. Castro ${ }^{3}$ \\ ${ }^{1}$ Instituto de Ciencia de Materiales de Madrid, CSIC, Cantoblanco, E-28049 Madrid, Spain \\ ${ }^{2}$ Condensed Matter Theory Group, Paul Scherrer Institute, CH-5232 Villigen PSI, Switzerland \\ ${ }^{3}$ CFIF, Instituto Superior Técnico, U Lisbon, Av. Rovisco Pais, 1049-001 Lisboa, Portugal
}

(Received 29 August 2013; revised manuscript received 22 October 2013; published 18 December 2013)

\begin{abstract}
We investigate the fate of interaction-driven phases in the half-filled honeycomb lattice for finite systems via exact diagonalization with nearest- and next-nearest-neighbor interactions. We find evidence for a charge density wave phase, a Kekulé bond order, and a sublattice charge-modulated phase in agreement with previously reported mean-field phase diagrams. No clear sign of an interaction-driven Chern insulator phase (Haldane phase) is found despite being predicted by the same mean-field analysis. We characterize these phases by their ground-state degeneracy and by calculating charge-order and bond-order correlation functions.
\end{abstract}

DOI: 10.1103/PhysRevB.88.245123

PACS number(s): 71.10.Hf, 71.27.+a

\section{INTRODUCTION}

The role of electron-electron interactions in graphene ${ }^{1}$ has been a fruitful subject of research even before this material was discovered. ${ }^{2}$ Although important progress has been made towards a full understanding of their effect, ${ }^{3}$ there are still fundamental questions that need to be clarified. One of such open questions regards the fate of band electrons in graphene's honeycomb lattice when subject to repulsive interactions at half-filling. The plethora of techniques ${ }^{4-8}$ available to study the effect of interactions in this system has produced a range of interesting predictions. In particular, in a series of works, ${ }^{4,5,9,10}$ several groups have produced compatible mean-field phase diagrams that suggest that electrons in a honeycomb lattice with extended Hubbard interactions at halffilling stabilize a Chern insulator (CI) phase with topological character and quantized Hall conductivity. This phase is nothing but the celebrated Haldane phase ${ }^{11}$ and it is realized at moderate values of the nearest-neighbor (NN) $V_{1}$ and nextto-nearest-neighbor (NNN) $V_{2}$ interactions but always with $V_{2}>V_{1}$.

Interestingly, the CI phase is embedded in a rich structure of other competing orders in the phase diagram. The first of these is a charge density wave ${ }^{4}$ order (CDW) at $V_{1} \gg V_{2}$ with charge imbalance between the two different sublattices that reduces the amount of NN interaction energy to be paid. (Long-range Coulomb interactions can affect this picture; see for example Ref. 12. ) A sublattice charge-modulated ${ }^{10}$ phase (CMs) was also found for $V_{2} \gg V_{1}$ with charge imbalance over the same sublattice that compensates for the large- $V_{2}$ cost. At sufficiently large $V_{2} \sim V_{1}$, a Kekulé bond order emerged ${ }^{9,13-16}$ characterized by a $Z_{3}$ order parameter which can lead to fractionalized excitations of $\pm e / 2$ at the long-wavelength limit. ${ }^{14}$ These phases have the additional interest of being also examples of an interaction-driven gap for low-energy quasiparticles in the honeycomb lattice. Together, these works provide a clear consistent picture of the possible phases available within the mean-field perspective. However, the results are subject to the limitations of mean-field theory, for (i) there is no certainty that all local order parameters relevant to the low-energy physics have been considered, ${ }^{10}$ (ii) the ground state of the system might not be adiabatically connected to mean-field state with a local order parameter, and (iii) the mean-field phase can be overestimated/underestimated in the parameter region of the phase diagram.

To test the mean-field picture it is necessary to employ different tools as independent checks for the presence of the mean-field phases. One of such tools is exact diagonalization (ED) which we explore in this work. It is based on the ED of the Hamiltonian for finite lattice sizes and it provides, in principle, an unbiased analysis of interactions. The main limitations for ED in two-dimensional quantum systems are the smallness of system sizes that can be studied. Finite-size effects might well out-range the energy scale of a potential many-body gap of incompressible ground states, so that the incompressibility can not be recognized. Therefore, the limitations of ED and the mean-field approach are to a large extent complementary. If both methods yield the same phase for a region in parameter space, this provides strong evidence that the true ground state in the thermodynamic limit will be of this nature. Phases that can not be easily detected with neither ED nor the mean-field approach include those with incommensurate long-range order. For example, depending on the system size and the particular geometry, ED might favor commensurate phases against frustrated phases and one has to be careful to explore (whenever possible) different sizes and/or aspect $\operatorname{ratios}^{17,18}$ to pin down the relevant competing phases. Indeed, ED has proven useful in studies of the Haldane-Hubbard model $^{17-19}$ and the $\pi$-flux model, ${ }^{20}$ complementing other techniques such as quantum Monte Carlo and variational cluster approximation used in studies of the Hubbard and Kane-Mele-Hubbard models in the honeycomb lattice. ${ }^{6,8,21-29}$

Motivated by these results, and in particular by the interaction-driven phases found in existing mean-field calculations, in this work we study the spinless extended Hubbard model with both NN and NNN interactions in the honeycomb lattice at half-filling via ED of small finite-size systems. We will investigate and characterize the phase diagram for electronic phases that are driven by Coulomb interactions in the honeycomb lattice as an independent check for the mean-field picture. We will provide evidence for the appearance of some of the phases that were previously obtained in mean-field calculations. These include the CDW, the Kekule bond order, and the CMs phases which surround a trivial semimetal (SM) phase. Surprisingly, for the studied lattice sizes, we find no clear sign of the previously reported interaction-driven CI 


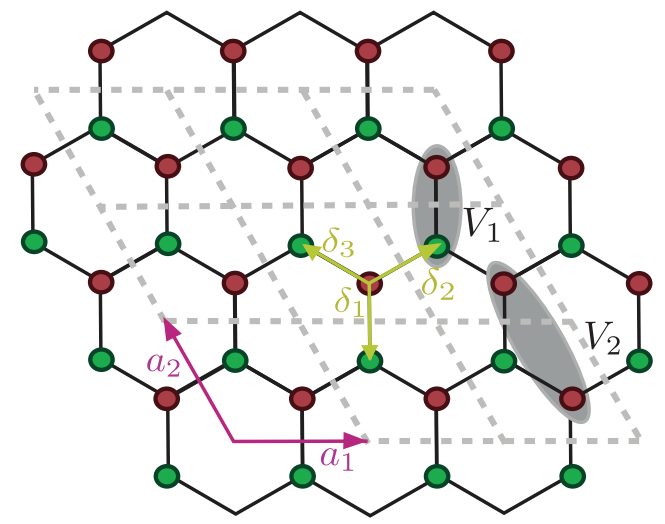

FIG. 1. (Color online) The extended Hubbard model on the honeycomb lattice. A and B sublattices are represented by green and red circles. The basis vectors and nearest-neighbors vectors defined in the text are $\boldsymbol{a}_{1,2}$ and $\boldsymbol{\delta}_{1,2,3}$, respectively. The NN and NNN interactions $V_{1}$ and $V_{2}$ are represented by gray ellipses. The gray dotted lines enclose the $\Omega=3 \times 3$ cluster with periodic boundary conditions.

phase. As for the phases that do appear, we will characterize them by their ground-state degeneracy and by computing the charge density and bond-order correlation functions.

In Sec. II, we introduce the model and establish notation conventions. In Sec. III, we present the complete phase diagram of the honeycomb lattice at half-filling with NN and NNN interactions. We will discuss the main properties and characterize each of the appearing phases. In Sec. IV, we relate our findings with previous works and discuss the absence of the interaction-driven CI phase. Finally, in Sec. V we summarize our main findings.

\section{MODEL}

We start with the spinless extended Hubbard model for electrons in a honeycomb lattice with nearest-neighbor (NN) interaction $V_{1}$ and next-to-nearest-neighbor (NNN) interaction $V_{2}$. The Hamiltonian in real space reads as

$$
H:=-t \sum_{\langle i, j\rangle}\left(c_{i}^{\dagger} c_{j}+\text { H.c. }\right)+V_{1} \sum_{\langle i, j\rangle} n_{i} n_{j}+V_{2} \sum_{\langle\langle i, j\rangle\rangle} n_{i} n_{j},
$$

where $t$ is the nearest-neighbor hopping and $c_{i}$ annihilates an electron at the $i$ th site of the honeycomb lattice. Each of the two triangular sublattices $\mathrm{A}$ and $\mathrm{B}$ is spanned by the basis vectors $\boldsymbol{a}_{1}=\boldsymbol{\delta}_{2}-\boldsymbol{\delta}_{3}$ and $\boldsymbol{a}_{2}=\boldsymbol{\delta}_{3}-\boldsymbol{\delta}_{1}$ defined through the three nearest neighbors $\delta_{1}=a(0,-1), \delta_{3}=a(\sqrt{3} / 2,1 / 2)$, and $\boldsymbol{\delta}_{3}=a(-\sqrt{3} / 2,+1 / 2)$ as shown in Fig. 1. Transforming to Fourier space by defining $a_{\boldsymbol{k}}^{\dagger}:=\frac{1}{\sqrt{\Omega}} \sum_{i \in A} c_{i}^{\dagger} e^{i \boldsymbol{k} \cdot \boldsymbol{r}_{i}}$ and $b_{\boldsymbol{k}}^{\dagger}:=$ $\frac{1}{\sqrt{\Omega}} \sum_{i \in B} c_{i}^{\dagger} e^{i \boldsymbol{k} \cdot \boldsymbol{r}_{i}}$, the Hamiltonian (1) can be expressed as

$$
\begin{aligned}
H= & -t \sum_{\boldsymbol{k}} \gamma_{\boldsymbol{k}} a_{\boldsymbol{k}}^{\dagger} b_{\boldsymbol{k}}+\text { H.c. } \\
& +\frac{V_{1}}{\Omega} \sum_{\boldsymbol{k}, \boldsymbol{k}^{\prime}, \boldsymbol{q}} \gamma_{\boldsymbol{q}} a_{\boldsymbol{k}}^{\dagger} a_{\boldsymbol{k}-\boldsymbol{q}} b_{\boldsymbol{k}^{\prime}}^{\dagger} b_{\boldsymbol{k}^{\prime}+\boldsymbol{q}}+\text { H.c. } \\
& +\frac{V_{2}}{\Omega} \sum_{\boldsymbol{k}, \boldsymbol{k}^{\prime}, \boldsymbol{q}} \chi_{\boldsymbol{q}}\left(a_{\boldsymbol{k}}^{\dagger} a_{\boldsymbol{k}-\boldsymbol{q}} a_{\boldsymbol{k}^{\prime}}^{\dagger} a_{\boldsymbol{k}^{\prime}+\boldsymbol{q}}+b_{\boldsymbol{k}}^{\dagger} b_{\boldsymbol{k}-\boldsymbol{q}} b_{\boldsymbol{k}^{\prime}}^{\dagger} b_{\boldsymbol{k}^{\prime}+\boldsymbol{q}}\right),
\end{aligned}
$$

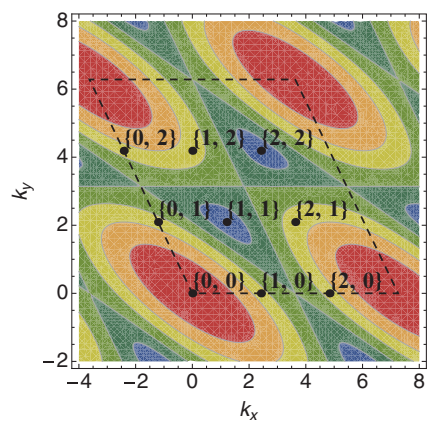

(a)

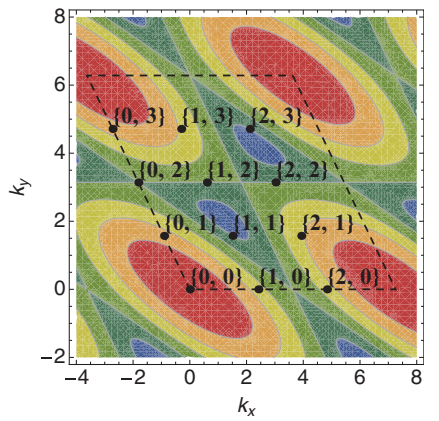

(b)
FIG. 2. (Color online) Contour plot of the noninteracting band structure obtained from (1) with $V_{1}=V_{2}=0$. Superimposed is the discretized BZ (dashed line) for a lattice with (a) $\Omega=3 \times 3$ and (b) $\Omega=3 \times 4$ unit cells. The inset text shows the momentum label $\boldsymbol{Q}=\left(Q_{1}, Q_{2}\right)$ (see text).

where $\quad \gamma_{\boldsymbol{k}}=\left(1+e^{i \boldsymbol{k} \cdot \boldsymbol{a}_{2}}+e^{i \boldsymbol{k} \cdot\left(\boldsymbol{a}_{1}+\boldsymbol{a}_{2}\right)}\right) \quad$ and $\quad \chi_{\boldsymbol{k}}=\left(e^{i \boldsymbol{k} \cdot \boldsymbol{a}_{1}}+\right.$ $\left.e^{i \boldsymbol{k} \cdot \boldsymbol{a}_{2}}+e^{i \boldsymbol{k} \cdot\left(\boldsymbol{a}_{1}+\boldsymbol{a}_{2}\right)}\right)$ are NN and NNN form factors, respectively, and $\Omega$ is the number of unit cells.

In what follows, we investigate the phase diagram as a function of $V_{1}$ and $V_{2}$ via $\mathrm{ED}$ of small clusters of size $\Omega=$ $3 \times 3$ (see Fig. 1) and $\Omega=3 \times 4$ using periodic boundary conditions.

\section{PHASE DIAGRAM}

In order to implement the ED of Hamiltonian (2), we discretize the Brillouin zone (BZ) with a lattice $\Omega=L_{1} \times L_{2}$ of points that span the BZ area. The band structure of graphene and two different sets of lattice sizes are shown in Fig. 2. For the $\Omega=3 \times 3$ lattice, there are nine points per band in the BZ each to be filled with one electron. There is one at the $\Gamma$ point, two at the $\boldsymbol{K}$ and $\boldsymbol{K}^{\prime}$ points, and a set of six energetically degenerate points. For a given particle $i$, we label its momentum by its coordinates in momentum space $k_{1}^{(i)}, k_{2}^{(i)}$ or, alternatively, with the discrete one-dimensional integer label $Q^{(i)}=k_{1}^{(i)}+L_{1} k_{2}^{(i)}$. In this notation and for this lattice, the $\Gamma$ point corresponds to momentum $(0,0)$, or $Q^{(i)}=0$, and the $\boldsymbol{K}$ and $\boldsymbol{K}^{\prime}$ points are at $(1,1)$, or $Q^{(i)}=4$, and $(2,2)$, or $Q^{(i)}=8$, respectively [see Fig. 2(a)]. In general, $k_{1}^{(i)} \in\left[0, L_{1}-1\right], k_{2}^{(i)} \in\left[0, L_{2}-1\right]$, and $Q^{(i)} \in\left[0, L_{1} L_{2}-1\right]$.

Since the interaction in Hamiltonian (2) conserves the total momentum, in ED we can diagonalize independently each total momentum sector subspace $Q=\sum_{i} Q^{(i)}$ with $Q \in$ $\left[0, L_{1} L_{2}-1\right]$, where the momentum is defined modulo $\Omega$. Therefore, all eigenvalues and eigenvectors that we obtain are labeled by $Q$. The phase diagram for $\Omega=3 \times 3$ with $N=9$ particles [i.e., $v=N /(2 \Omega)=\frac{1}{2}$ filling] and representative eigenvalue spectra as a function of $Q$ are shown in Fig. 3(i). By focusing on the ground-state degeneracy, we identify a phase by the number of ground states over which there is the highest gap. We will thus define for each phase the quasidegenerate ground state as the set of $n$ states that are lowest in energy if the gap above these $n$ states is larger than the gap above any other set of $n^{\prime} \neq n$ lowest states. In what follows, we will distinguish and characterize the four distinct phases. We will 


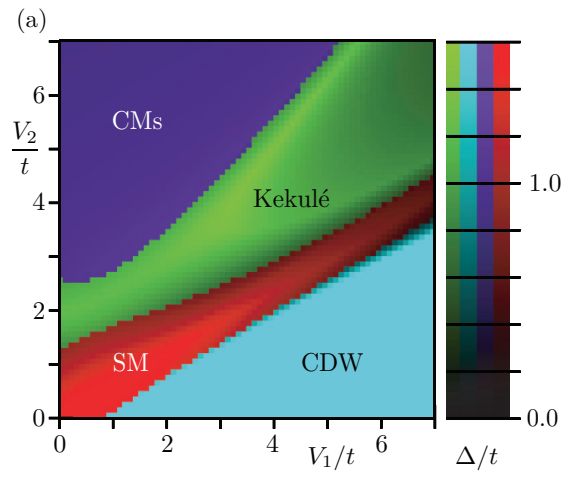

(i) $\begin{array}{ll}\text { (b) CMs, } V_{1}=0, V_{2}=6 & \text { (c) Kekulé, } V_{1}=V_{2}=3\end{array}$

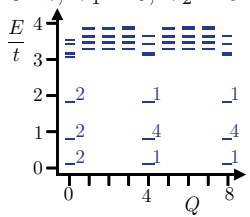

(d) SM, $V_{1}=V_{2}=0$

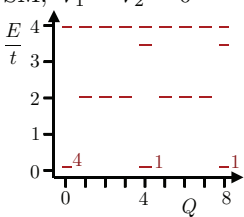

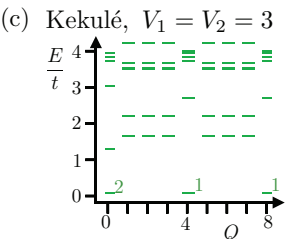

(e) CDW, $V_{1}=2, V_{2}=0$

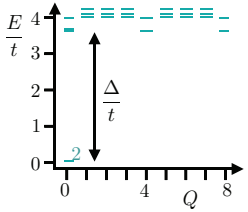

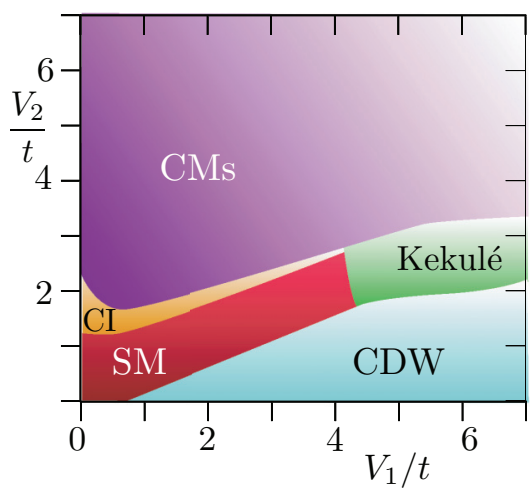

(ii)

FIG. 3. (Color online) (i) ED phase diagram at $n=\frac{1}{2}$ for a $\Omega=3 \times 3$ system. The brightness of each color is proportional to the size of the many-body gap $\Delta / t$. The right-hand side shows the energy spectrum against total momentum $Q=Q_{1}+L_{1} Q_{2}$ for the (b) CMs phase, (c) Kekule phase, (d) SM phase, and (e) CDW phase. The small numbers indicate the degeneracy of each state. The zero of energies is chosen to be the ground-state energy. The phases are identified by the number of ground states over which there is the highest gap. (ii) Mean-field phase diagram calculated following Ref. 10.

argue that they correspond to the SM, Kekulé, CDW, and CMs phases and discuss their main signatures. We define a phase transition when the gap above the number of ground states on either side of the transition is equal. We note that the phase boundaries might be altered by going to larger systems or applying alternative definitions to identify the phases.

In the following section, we will use these findings to relate to previous works to finally compare with the mean-field diagram in Fig. 3(ii) of Ref. 10 which includes all possible (nonsuperconducting) mean-field decouplings with a tripled unit cell. In particular, this phase diagram is consistent with past mean-field studies for which the absence of the Kekulét,7 or CMs phases ${ }^{4,5,7,9}$ in the mean-field phase diagrams was due to the fact that these works did not allow for these mean-field solutions.

\section{A. Semimetal phase}

This phase, labeled SM and shown in red in Fig. 3(a), is straightforward to characterize since it stems from the noninteracting ( $V_{1}=V_{2}=0$ ) limit of Hamiltonian (2). For $\Omega=3 \times 3$ at half-filling $(N=9)$, there are $2 \Omega=18$ lattice sites to fit 9 particles. Seven of them sit at the lower states, one at the $\Gamma$ point at $(0,0)$, and six particles go to the six degenerate momenta at $(1,0),(2,0),(0,1),(2,1),(0,2),(1,2)$. We have two particles left for four degenerate single-particle states, two at the $\boldsymbol{K}$ point and two at the $\boldsymbol{K}^{\prime}$ point, since at these points there are two degenerate states, one from each band. This gives a freedom to choose the ground state. We have two particles to fill four states, the degeneracy of which is given by the binomial coefficient $C(4,2)=6$ which is the ground-state degeneracy for the noninteracting case. Out of these six possibilities, four of them have a particle at $\boldsymbol{K}$ and a particle at $\boldsymbol{K}^{\prime}$ and thus a total momentum of $Q=0$. The remaining two configurations have two particles at the same valley. Having both at $\boldsymbol{K}=(1,1)$ results in a total momentum $(2,2)$ or $Q=8$. Similarly placing the two last particles at $\boldsymbol{K}^{\prime}=(2,2)$ we expect to have a single state at momentum $(1,1)$, or $Q=4$.
To summarize, the noninteracting Hamiltonian in ED has a sixfold-quasidegenerate ground state at half-filling with four states at $Q=0$, one state at $Q=4$ and one state at $Q=8$.

We observe this structure for a finite region of parameters colored red in Fig. 3(a) connected to the noninteracting Hamiltonian and thus we interpret this phase as a SM phase. The spectrum for such phase is shown in Fig. 3(d) where the sixfold-quasidegenerate ground state is observed at the momenta discussed above. The spectral evolution of the energy levels within the phase upon changing $V_{1}$ and $V_{2}$ is smooth with no level crossings.

Finally, note that the SM phase has all discrete symmetries, i.e., time reversal $(\mathcal{T})$, inversion $(\mathcal{I})$, and the combined action of both $(\mathcal{T} \mathcal{I})$. Out of the sixfold-quasidegenerate ground states, the states at $Q=\boldsymbol{K}, \boldsymbol{K}^{\prime}$ would interchange under the action of the operators representing $\mathcal{T}, \mathcal{I}$. The operation $\mathcal{T} \mathcal{I}$ leaves the momentum quantum numbers invariant.

\section{B. Charge density wave phase}

The second phase that we identify is labeled CDW and is shown in light blue in Fig. 3(a). Its spectrum shows a twofold-degenerate ground state at $Q=0$ [Fig. 3(e)] and would break spontaneously the sublattice symmetry in the thermodynamic limit. The most transparent way to understand that this phase is indeed a CDW is to investigate the strong coupling limit at $V_{1} / t \rightarrow \infty$ with $V_{2}=0$ to which this phase is adiabatically connected. Calculating the degeneracy of such a strong coupling state is a classical problem, the ground state of which is represented in Fig. 4.

As only one sublattice is occupied in either of these classical ground states, both of them are zero-energy eigenstates of the NN interaction $V_{1}$. We expect this state to appear at total momentum $Q=0$ since it is a charge density wave (CDW) order state within the unit cell. Indeed, the ED of the Hamiltonian with $V_{1} \neq 0$ and $V_{2}=t=0$ yields exactly this twofold-degenerate ground state at zero energy. The excited 


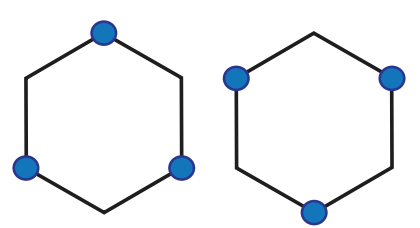

FIG. 4. (Color online) CDW pattern at $V_{1} / t \rightarrow \infty$ with $V_{2}=0$.

states that can be computed classically also coincide both in energy and degeneracy in this limit.

It is possible to connect this strong coupling phase to the twofold-degenerate phase shown in Fig. 3 simply by increasing the hopping continuously to see that both phases are indeed connected without ever closing the many-body gap. From this fact alone we can already conclude that this state is a CDW state. A further check of this picture comes from calculating the charge density wave modulation in the same spirit as described in Ref. 30. Suppose that we have a phase with a set of (quasi)degenerate ground states $|m\rangle, m=1, \ldots, N_{\mathrm{gs}}$. For these, we define the sublattice-staggered electron density matrix

$$
\begin{aligned}
\rho_{r}^{m m^{\prime}}:= & \frac{1}{\Omega} \sum_{k} e^{i\left(Q_{m}-Q_{m^{\prime}}\right) \cdot r} \\
& \times\left\langle m\left|a_{k+\left(Q_{m}-Q_{m^{\prime}}\right)}^{\dagger} a_{k}-b_{k+\left(Q_{m}-Q_{m^{\prime}}\right)}^{\dagger} b_{k}\right| m^{\prime}\right\rangle .
\end{aligned}
$$

For the CDW case, we have in particular that $m=1,2$ with both $\boldsymbol{Q}_{1}=\boldsymbol{Q}_{2}=0$. Note that since $\boldsymbol{Q}_{1}=\boldsymbol{Q}_{2}$ it is not possible to build a linear combination of ground states that generates charge modulation outside the unit cell which is consistent with the CDW we are trying to characterize.

We now diagonalize the $2 \times 2$ matrix $\rho_{r}$ for representative points inside the CDW phase. This generates two $r$ independent eigenvectors and eigenvalues $v_{i}$ and $\lambda_{i}(i=1,2)$. The former represent the two independent superpositions of the twofold-degenerate ground states while the latter represent the two possible sublattice imbalances. Therefore, if finite, the eigenvalues are the defining feature of the CDW phase. For example, for $V_{1}=5 t$ and $V_{2}=0$ we find that $\lambda_{1}=-0.99$ and $\lambda_{2}=0.97$. The former (latter) corresponds to a state with particles localized in sublattice B (A) as represented in Fig. 4 confirming the CDW interpretation of the state.

Finally, a transparent way to understand this state is to relate it with the noninteracting honeycomb lattice with a staggered chemical potential $\pm m$ in the A (B) sublattice, i.e., the noninteracting version of the CDW state. Upon filling the band structure for this simple case at half-filling and for $\Omega=3 \times 3$, the two particles highest in energy have momenta $\boldsymbol{K}$ and $\boldsymbol{K}^{\prime}$, thus corresponding to a single state of total momentum $Q=0$. Within the interacting model and since symmetry breaking is absent for finite systems, by ED of the interacting Hamiltonian we find both $\pm m$ and $\mp m$ configurations which then give a degeneracy of two. Under $\mathcal{I}$, the two ground states of the CDW interchange. This is probed by the order parameter (3), specifically designed to test this symmetry for the two quasidegenerate ground states.

\section{Kekulé phase}

The next phase that we identify is labeled the Kekule phase shown in green color in Fig. 3(a). It has a fourfold-degenerate ground state [Fig. 3(c)] at $Q=0(\times 2), 4,8$ corresponding to two states at the $\boldsymbol{\Gamma}$ point and one state at both $\boldsymbol{K}$ and $\boldsymbol{K}^{\prime}$. As mentioned above, ED of finite systems can only yield precursors of spontaneous symmetry breaking in the thermodynamic limit. This means that if the Kekule order is present, it should appear in all of its linearly independent forms which can be naively counted to be six, depicted in Fig. 5(i). However, of these six possible Kekulé orders, three for each NN hopping distortion $t \pm \delta t$, only four are linearly independent, and the remaining two can be obtained as linear combinations of the other four [see caption in Fig. 5(i)]. For example, from the six patterns in Fig 5(i) we can produce the second row out of the first row only if we use an overall, homogeneous decrease in hopping. This particular shift one can interpret as a linear combination of the second row, i.e., (d) $+(\mathrm{e})+(\mathrm{f})$. Therefore, the first row plus a linear homogeneous combination of the second row produces all states in the second row and so only four states are independent. The Kekulé bond order is therefore a candidate phase to explain the apparent fourfold-degenerate ground states in some region of the phase diagram [see Fig. 3(c)]. The fact that the momenta appear at $\boldsymbol{\Gamma}, \boldsymbol{K}$, and $\boldsymbol{K}^{\prime}$ supports this scenario since for a unit cell three times larger they all fold into the $\Gamma$ point, which means that one can build a hopping perturbation with a periodicity of three unit cells, exactly as the Kekulé would need.

In order to further evidence that this is a Kekulé phase, we explore a construction similar to the charge density wave matrix (3) this time for the hopping amplitudes

$$
t_{r}^{m m^{\prime}}=\frac{1}{\Omega} \sum_{k}\left\langle m\left|a_{k+\left(Q_{m}-Q_{m^{\prime}}\right)}^{\dagger} b_{k}\right| m^{\prime}\right\rangle e^{i\left(Q_{m}-Q_{m^{\prime}}\right) \cdot r}
$$

with the same notation as above but now for $m=1,2,3,4$ quasidegenerate ground states. In this case, the momentum differences $\boldsymbol{Q}_{m}-\boldsymbol{Q}_{m^{\prime}} \in\left\{0, \boldsymbol{K}, \boldsymbol{K}^{\prime}\right\}$ allow for a Kekulé bond order. As before, we diagonalize the matrix (4) and label the system of four eigenvalues and eigenvectors $\lambda_{m}$ and $v_{r}^{(m)}$. This time, the eigenvectors depend on position. If present, the Kekule bond order will appear as a superposition of the allowed phase factors $e^{i\left(\boldsymbol{Q}_{m}-\boldsymbol{Q}_{m^{\prime}}\right) \cdot \boldsymbol{r}}$. We can construct four independent superpositions corresponding to the four eigenvectors such that

$$
t_{\boldsymbol{r}}^{m}=2 \cos \left(v_{\boldsymbol{r}, 1}^{(m)}+v_{\boldsymbol{r}, 2}^{(m)}+v_{\boldsymbol{r}, 3}^{(m)}+v_{\boldsymbol{r}, 4}^{(m)}\right)
$$

with $m=1,2,3,4$. When evaluated at the three different links $t_{\boldsymbol{r}}^{m}, t_{\boldsymbol{r}+a_{1}}^{m}, t_{\boldsymbol{r}+a_{2}}^{m}$, the underlying hopping lattice of this phase is revealed. There are four of such patterns, one for each value of $m$.

However, if in the Kekulé phase, this procedure will in general produce an arbitrary superposition of all the possible Kekule structures of Fig. 5(i). The four independent superpositions are shown in Fig. 5(ii) where the three different colors represent different bond strengths. Note that each of these patterns has a tripled unit-cell periodicity with the right Kekule orders, inherited from the $\boldsymbol{r}$-dependent vectors $\boldsymbol{v}_{\boldsymbol{r}}^{(m)}$. Indeed, by forming linear combinations of these, one can obtain all the "pure" (coherent) Kekulé patterns in Fig. 5(i). 


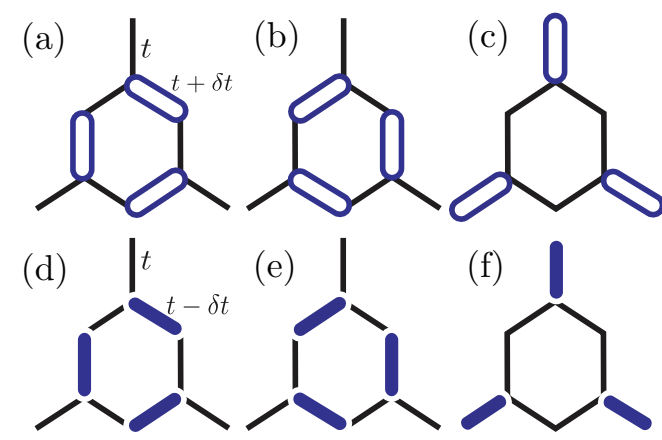

(i)

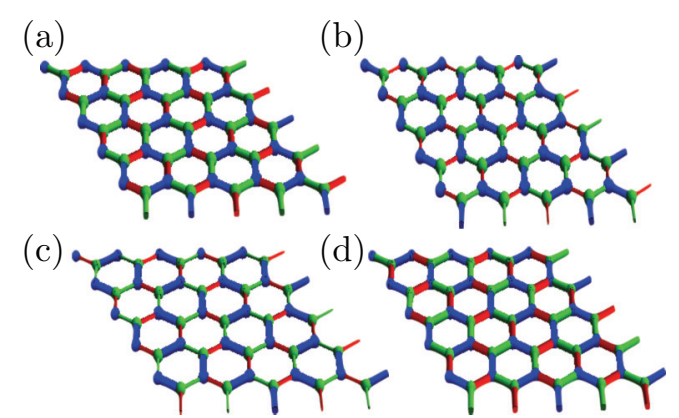

(ii)

FIG. 5. (Color online) (i) The six different types of Kekulé orders. From these six patterns one can produce the second row out of the first row only by including an overall homogeneous hopping decrease $(\mathrm{d})+(\mathrm{e})+(\mathrm{f})$. Thus, only four patterns are independent. (ii) Four independent Kekulé super-positions obtained from (5) with $V_{1}=3 t$ and $V_{2}=2 t$. Different colors correspond to different hopping magnitudes.

We find the analysis of this section to be consistent with the presence of the Kekule phase in this part of the phase diagram.

\section{Sublattice charge modulation (CMs)}

We finally address the last phase that remains to be characterized which we shall name as sublattice charge modulation or CMs appearing at the upper left corner of the phase diagram in Fig. 3(a). This phase, unlike the CDW, does not correspond to the naive classical strong coupling phase in the corresponding strong coupling limit. Rather, the limit $V_{2} / t, V_{2} / V_{1} \rightarrow \infty$ has an extensive classical ground-state degeneracy so that quantum corrections will determine the form of the actual ground state for arbitrarily small nonzero values of $t / V_{2}$ and $V_{1} / V_{2}$. The classical counting yields a 666 -fold-degenerate ground state with energy $18 V_{2}$ for a $\Omega=3 \times 3$ lattice. This information serves in fact as a consistency check just as in the CDW case. Indeed, we recover numerically the correct degeneracy and ground-state energy in the limit $V_{1} / t \rightarrow 0$ and $V_{2} \neq 0$.

The question then becomes what phase will be selected by the quantum fluctuations out of the classical groundstate manifold. From a large system with periodic boundary conditions, a natural phase to be expected at half-filling for large $V_{2} / V_{1}$ is that with a charge modulation within the same sublattice, the CMs phase. This phase, discussed in detail in Ref. 10 , reduces $V_{2}$ by paying an additional $V_{1}$ cost. Pictorially, the state is shown in Fig. 6 where it is evident that it has a degeneracy of 18 because of the rotational symmetry of the Hamiltonian. We find a phase consistent with this picture at large $V_{2} / V_{1}$ in ED with a quasidegeneracy of 18. Such a degeneracy slowly becomes more exact as one increases $V_{2} / V_{1}$, although the gap to the excited states also

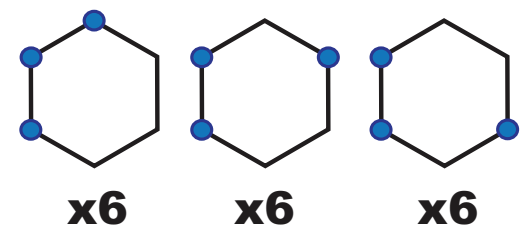

FIG. 6. (Color online) CMs patterns with their corresponding degeneracies due to a sixfold-rotational symmetry. decreases such that in the limit $V_{1} / t \rightarrow 0$ and $V_{2} \neq 0$ the strong coupling phase is recovered. Note also that, just as the Kekulé, the CMs state has also a tripled unit-cell periodicity ${ }^{10}$ which is consistent with having the ground states at momenta $\boldsymbol{Q} \in\left\{0, \boldsymbol{K}, \boldsymbol{K}^{\prime}\right\}$.

\section{DISCUSSION AND ABSENCE OF THE CI PHASE}

Despite the small system size, it is remarkable that the phase diagram Fig. 3(i) resembles very closely the mean-field phase diagram of Ref. 10 at half-filling shown in Fig. 3 (ii). We have found, out of the five mean-field phases, all but the CI phase. It is worth pointing out also that we do not observe level crossing. Rather, a ground-state manifold smoothly evolves into another as $V_{1,2}$ are varied.

The $\Omega=3 \times 3$ lattice studied above is special in that not only it contains the $\boldsymbol{K}$ and $\boldsymbol{K}^{\prime}$ which enables clear physical interpretation of the emerging phases, but also it fits phases with a tripled unit cell without frustration, such as the Kekulé or the CMs phases. Therefore, such a lattice size has a natural bias towards these phases as compared to the CI phase, which does not break translational symmetries. This might be the reason why the Kekule phase is so prevalent as compared to the mean-field phase diagram in Fig. 3(ii). It is also interesting to note that the Kekule phase shifts to higher values of $V_{2}$ reducing the region for the charge-modulated phase when comparing with the mean-field result. Since both phases are favored by the $\Omega=3 \times 3$ lattice size, this result seems robust.

To investigate further the presence of the CI phase, we have studied the $\Omega=3 \times 4$ and $4 \times 3$ lattices for $V_{1}=0$, where the CI phase is expected to appear from the mean-field analysis at intermediate $V_{2}$. These lattice sizes frustrate the Kekulé and can leave phase space for other phases (such as the CI phase) to appear.

The CI phase for a finite system would appear as a twofold-quasidegenerate ground state, one for each sign of the flux, in a similar way as the CDW shows a twofold-degenerate ground state corresponding to $\pm m$ or $\mp m$ charge in the A and B sublattices. Each state independently is invariant under $\mathcal{I}$, but not under $\mathcal{T}$ or $\mathcal{T} \mathcal{I}$. The latter two will map one state into the other. Aside from this signature, it is important to 
emphasize that the CI phase is not trivial to characterize in exact diagonalization. For instance, a naive calculation of the Chern number will always give zero since we would have a superposition of two CI phases that are related by reverting the sign of the flux. In addition, time reversal which is relevant to this phase is not a unitary symmetry and thus we can not diagonalize the operator that represents it in the basis of the ground-state manifold. An alternative possibility is to interpolate between the Haldane Hamiltonian ${ }^{11}$ and the interacting honeycomb lattice model studied in this paper to see if any of the phases is adiabatically connected to the Haldane's CI phase.

However, we have found no evidence of a twoquasidegenerate ground state and thus we conclude that this phase is absent also from the ED of $\Omega=3 \times 4$ and $4 \times 3$ lattices. As for the the $3 \times 3$ lattice, we have argued in past sections that all numerical results can be explained consistently without the need of a CI phase. For the $3 \times 4$ lattice, the spectra along the $V_{2}$ line shows first a single ground state at $Q=6$ for low $V_{2}$, as expected for the trivial SM phase just by adding all the noninteracting momenta in Fig. 2(b). At $V_{2} \sim 7 t$, the gap closes and reopens with a sixfold-quasidegenerate ground state. Despite that the lowest pair of these states lies at $Q=6$, as would be expected for the CI phase, the gap with the other four states is so small that it is unreasonable to interpret them as the finite-size precursor of a gapped ground-state manifold of the system. It remains an open question as to whether this sixfold degeneracy becomes twofold by increasing the lattice size, which could in principle lead in the thermodynamic limit to the appearance of the Haldane phase.

Finally, we comment on a different route towards achieving interaction-driven topological phases in the extended Hubbard model on the honeycomb lattice which involves moving away from half-filling. An example of these topological phases was shown to appear at higher fillings from a mean-field calculation in Refs. 5 and 10. These are generalizations of the Haldane phase at fillings $v \gtrsim \frac{2}{3}$ and with a tripled unit cell, which could also be present via ED. However, identifying these phases by characterizing the ground-state properties from ED is challenging due to band folding.

\section{CONCLUSIONS}

We have investigated the effect of extended Hubbard interactions on spinless electrons on the honeycomb lattice at half-filling via exact diagonalization (ED). We have found that four out of the five predicted mean-field phases are present.
These are the semimetal (SM), CDW, the Kekule bond order, and the sublattice charge modulation (CMs) phases. First, we have shown that the sixfold degeneracy of the SM ground state can be understood entirely from the noninteracting band structure. For the CDW phase, we have proven that it is connected to the strong coupling phase at $V_{1} / t \rightarrow \infty, V_{2}=0$ and we have characterized it finding a finite sublattice charge imbalance through the charge order correlation function, the hallmark for the CDW phase. The twofold degeneracy is a sign of the two possible orders that the system can choose in the thermodynamic limit by spontaneously breaking the sublattice symmetry. Similarly, we have disentangled the Kekulé bond-order phase by calculating the bond-order correlation function which reveals the underlying superposition of four independent Kekulé patterns which conform the fourfoldquasidegenerate ground state. Finally, we have argued that for $V_{2}>V_{1}$ the CMs phase is expected to have an 18-fold degeneracy favored by the costly NNN interaction, which is consistent with what we observed in ED.

Importantly, the fact that the discussed phases appear both in ED and in mean field suggests that they are stable up to the thermodynamic limit. The appearance of the Kekule phase dominating a wide region of the phase diagram opens up the possibility of realizing this exotic phase in cold atoms with a scheme along the lines of Ref. 7. Despite the fact that we have not found evidence for the Chern insulator (CI) phase, it is still possible that it is realizable in the thermodynamic limit. Different approaches such as cluster mean field ${ }^{31}$ can also prove useful to ascertain the presence of the CI phase. We hope that the conclusions of this work will motivate further explorations of the extended Hubbard model on the honeycomb lattice.

Note added. Recently, we learned from a complementary analysis $^{31}$ that focuses on the line $V_{2} \neq 0, V_{1}=0$ in the phase diagram. The results are consistent with those presented here, in particular, with the absence of the CI phase and the appearance of the CMs state.

\section{ACKNOWLEDGMENTS}

We acknowledge conversations with M. A. H. Vozmediano, F. de Juan, A. Cortijo, and C. Mudry. We also thank M. Hohenadler and M. Daghofer for sharing their results prior to publication. This research was supported in part by the Spanish MECD Grants No. FIS2011-23713, No. FIS201129689, No. PIB2010BZ-00512, and by the Swiss National Science Foundation.
${ }^{1}$ A. H. C. Neto, F. Guinea, N. M. R. Peres, K. S. Novoselov, and A. K. Geim, Rev. Mod. Phys. 81, 109 (2009).

${ }^{2}$ J. González, F. Guinea, and M. A. H. Vozmediano, Nucl. Phys. B 424 [FS], 595 (1994).

${ }^{3}$ V. N. Kotov, B. Uchoa, V. M. Pereira, F. Guinea, and A. H. Castro Neto, Rev. Mod. Phys. 84, 1067 (2012).

${ }^{4}$ S. Raghu, X.-L. Qi, C. Honerkamp, and S.-C. Zhang, Phys. Rev. Lett. 100, 156401 (2008).
${ }^{5}$ E. V. Castro, A. G. Grushin, B. Valenzuela, M. A. H. Vozmediano, A. Cortijo, and F. de Juan, Phys. Rev. Lett. 107, 106402 (2011).

${ }^{6}$ M. Hohenadler, Z. Y. Meng, T. C. Lang, S. Wessel, A. Muramatsu, and F. F. Assaad, Phys. Rev. B 85, 115132 (2012).

${ }^{7}$ A. Dauphin, M. Muller, and M. A. Martin-Delgado, Phys. Rev. A 86, 053618 (2012).

${ }^{8}$ H.-H. Hung, V. Chua, L. Wang, and G. A. Fiete, arXiv:1307.2659.

${ }^{9}$ C. Weeks and M. Franz, Phys. Rev. B 81, 085105 (2010). 
${ }^{10}$ A. G. Grushin, E. V. Castro, A. Cortijo, F. de Juan, M. A. H. Vozmediano, and B. Valenzuela, Phys. Rev. B 87, 085136 (2013).

${ }^{11}$ F. D. M. Haldane, Phys. Rev. Lett. 61, 2015 (1988).

${ }^{12}$ J. González, J. High Energy Phys. 07 (2013) 175.

${ }^{13}$ C. Chamon, Phys. Rev. B 62, 2806 (2000).

${ }^{14}$ C.-Y. Hou, C. Chamon, and C. Mudry, Phys. Rev. Lett. 98, 186809 (2007).

${ }^{15}$ B. Roy and I. F. Herbut, Phys. Rev. B 82, 035429 (2010).

${ }^{16}$ B. Roy, V. Juricic, and I. F. Herbut, Phys. Rev. B 87, 041401 (2013).

${ }^{17}$ C. N. Varney, K. Sun, M. Rigol, and V. Galitski, Phys. Rev. B 82, 115125 (2010).

${ }^{18}$ C. N. Varney, K. Sun, M. Rigol, and V. Galitski, Phys. Rev. B 84, 241105 (2011).

${ }^{19}$ L. Wang, H. Shi, S. Zhang, X. Wang, X. Dai, and X. C. Xie, arXiv:1012.5163.

${ }^{20}$ Y. Jia, H. Guo, Z. Chen, S.-Q. Shen, and S. Feng, Phys. Rev. B 88, 075101 (2013).
${ }^{21}$ S. Rachel and K. Le Hur, Phys. Rev. B 82, 075106 (2010).

${ }^{22}$ Z. Y. Meng, T. C. Lang, S. Wessel, F. F. Assaad, and A. Muramatsu, Nature (London) 464, 847 (2010).

${ }^{23}$ M. Hohenadler, T. C. Lang, and F. F. Assaad, Phys. Rev. Lett. 106, 100403 (2011).

${ }^{24}$ D. Zheng, G.-M. Zhang, and C. Wu, Phys. Rev. B 84, 205121 (2011).

${ }^{25}$ S.-L. Yu, X. C. Xie, and J.-X. Li, Phys. Rev. Lett. 107, 010401 (2011).

${ }^{26}$ W. Wu, S. Rachel, W.-M. Liu, and K. Le Hur, Phys. Rev. B 85, 205102 (2012).

${ }^{27}$ S. Sorella, Y. Otsuka, and S. Yunoki, Sci. Rep. 2, 992 (2012).

${ }^{28}$ H.-H. Hung, L. Wang, Z.-C. Gu, and G. A. Fiete, Phys. Rev. B 87, 121113 (2013)

${ }^{29}$ B. K. Clark, arXiv:1305.0278.

${ }^{30}$ A. G. Grushin, T. Neupert, C. Chamon, and C. Mudry, Phys. Rev. B 86, 205125 (2012).

${ }^{31}$ M. Daghofer and M. Hohenadler, arXiv:1308.6211. 